\title{
A novel error critic for variable structure control with an ADALINE
}

\section{Mehmet Önder Efe}

Electrical and Computer Engineering Department, Carnegie Mellon University, 5000 Forbes Avenue, Pittsburgh, PA 15213-3890, USA

\begin{abstract}
This paper discusses a novel method for variable structure control. The method aims to create and maintain a predefined sliding motion in the phase space by adjusting the parameters of an ADALINE-type controller. The objective of the paper is to demonstrate that the task can be achieved under the assumption that the plant under control could be driven towards the sliding manifold. The approach presented assumes the structure of the dynamic representation of the plant is known but the analytical details embodying it are unavailable. The results obtained confirm the prescribed form of tracking claim together with low computational cost due to the adjustment of the controller parameters.
\end{abstract}

Key words: ADALINE; control error extraction; parameter tuning; sliding mode control.

\section{Introduction}

It is a well known fact that a variable structure controller with a switching output drives the error vector towards the origin in two phases. These are namely the reaching phase, which lasts until the error vector hits the sliding manifold, and the sliding phase, during which the error vector converges to the origin as characterized by the locus along the sliding hypersurface. Provided that the uncertainties satisfy the matching conditions, the control system becomes totally insensitive to the disturbances during the sliding mode, and the extent of this robust behaviour is determined by the physical constraints, such as the actuation speed of the plant

Address for correspondence: M.Ö. Efe, Electrical Engineering Department, The Ohio State University, 205, Dreese Laboratories, 2015 Neil Avenue, Columbus, OH 43210, USA. E-mail: onderefe@ieee.org 
under control or the bounds on the applicable control signals. The strategy is therefore known as sliding mode control (SMC) in the related literature.

The studies, which report the use of the SMC technique for parameter tuning in flexible structures, by Sanner and Slotine (1992), and Sira-Ramirez and ColinaMorles (1995) have been the stimulants proving that the robustness feature of SMC could be exploited in parameter tuning. These studies pioneered a vast majority of researchers working on SMC-based parameter tuning. Sanner et al. (1992) have considered the training of Gaussian radial basis function neural networks (GRBFNN), which have certain degrees of analytical tractability in explaining the stability issues, and Sira-Ramirez et al. (1995) have shown the use of adaptive linear elements (ADALINE) with an SMC-based learning strategy. As an illustrative example, the inverse dynamics identification of a Kapitsa pendulum has been demonstrated, together with a thorough analysis towards the handling of disturbances. Hsu and Real $(1997,1999)$ demonstrate the use of SMC with GRBFNN; Yu et al. (1998) introduce the dynamic uncertainty adaptation of what is proposed by Sira-Ramirez et al. (1995), and demonstrate the performance of the scheme on the Kapitsa pendulum. Parma et al. (1998) use the SMC technique in the parameter tuning process of a multilayer perceptron. The latest studies towards the integration of SMC and parameter tuning mechanisms have shown that the tuning can be implemented on dynamic weight filter neurons (Sira-Ramirez et al., 2000), and on the parameters of a controller (Efe et al., 2000). An extensive review is provided in the recent survey of Kaynak et al. (2001). The survey illustrates how SMC can be used for parameter adjustment in computational intelligence.

A substantial problem in utilizing the SMC-based tuning strategies for control applications is the lack of the error on the output of the controller. This is primarily due to the unavailability of the target values of the controller outputs. When this problem is considered with control of uncertain plants, the tuning of the controller parameters becomes a challenge due to the information-limited nature of the problem. The major contribution of this paper is the illumination of this problem for a class of nonlinear systems with the assumption that SMC task is achievable. In the second section, the structure of the control system is explained; the section following this briefly introduces the conventional SMC technique. The fourth section is devoted to the calculation of the control error, and the fifth section describes the parameter adaptation strategy. In the sixth section, we discuss several issues related to the practical implementations of the proposed approach. The seventh section presents an illustrative example and conclusions constitute the last part of the paper.

\section{Control system structure}

Consider the nonlinear time-varying system given in (1). The system is composed of $n$ subsystems, the $i$ th one of which is of order $r_{i}$. The state vector of the system is $\underline{\theta}=\left[\theta_{1} \theta_{1} \ldots \theta_{1}^{\left(r_{1}-1\right)} \ldots \theta_{n} \breve{\theta}_{n} \ldots \theta_{n}^{\left(r_{n}-1\right)}\right]^{T}$ and the input vector is $\underline{\tau}=\left[\begin{array}{lll}\tau_{1} & \tau_{2} \ldots \tau_{n}\end{array}\right]^{T}$.

$$
\theta_{i}^{\left(r_{i}\right)}=f_{i}(\underline{\theta}, t)+\sum_{j=1}^{n} d_{i j}(t) \tau_{j} \quad i=1,2, \ldots, n
$$


Denoting $m=\sum_{i=1}^{n} \dot{r}_{i}$, the system of (1) with these vectors can more compactly be restated as $\underline{\dot{\theta}}=\underline{F}(\underline{\theta}, t)+D(t) \tau$, where $\underline{F}(\theta, t)$ is $m \times 1$ and $D(t)$ is $m \times n$ dimensional. It is assumed that the functions $\mathrm{f}_{i}(\underline{\theta}, t)$ are unknown and therefore the $n$ out of $m$ entries of vector function $\underline{F}(\underline{\theta}, t)$ are unavailable, whereas $d_{i j} \mathrm{~s}$ (or consequently the corresponding entries of $D(t)$ ) are assumed to be available. The design problem is to enforce the response of the system towards the desired trajectory $\left(\underline{\theta}_{d}\right)$, which is known but the control signal resulting in which is unavailable since $\underline{F}(\theta, t)$ is not known explicitly. Therefore, the solution to this problem is a search towards the synthesis of such a signal iteratively by a learning controller. Assuming that the controller in Figure 1 is composed of $n$ individual subcontrollers, the $i$ th one of which is to construct the $i$ th component of input vector $\underline{\tau}$. The $j$ th entry of the excitation vector driving this subcontroller can be given as $e_{i}^{(j)}=\theta_{i}^{(j)}-\theta_{d_{i}}^{(j)}$. Apparently, this component is the $j$ th derivative of the $i$ th tracking error component $\left(e_{i}\right)$.

\section{Conventional SMC design - an overview}

Consider the vector of sliding surfaces for the system in (1): $\underline{s}(\underline{e})=G \underline{e}$, where $\underline{e}=\underline{\theta}-\underline{\theta}_{d}$ is the tracking error vector and $G$ is a $n \times m$ dimensional design matrix describing the vector of sliding surfaces. The widespread selection of the matrix $G$ is such that the $i$ th sliding surface function has the form

$$
s_{i}\left(e_{i}\right)=\left(\frac{d}{d t}+\lambda_{i}\right)^{r_{i-1}} e_{i}
$$

in which $\lambda_{i}$ is a strictly positive constant. Let $V$ be a candidate Lyapunov function given as

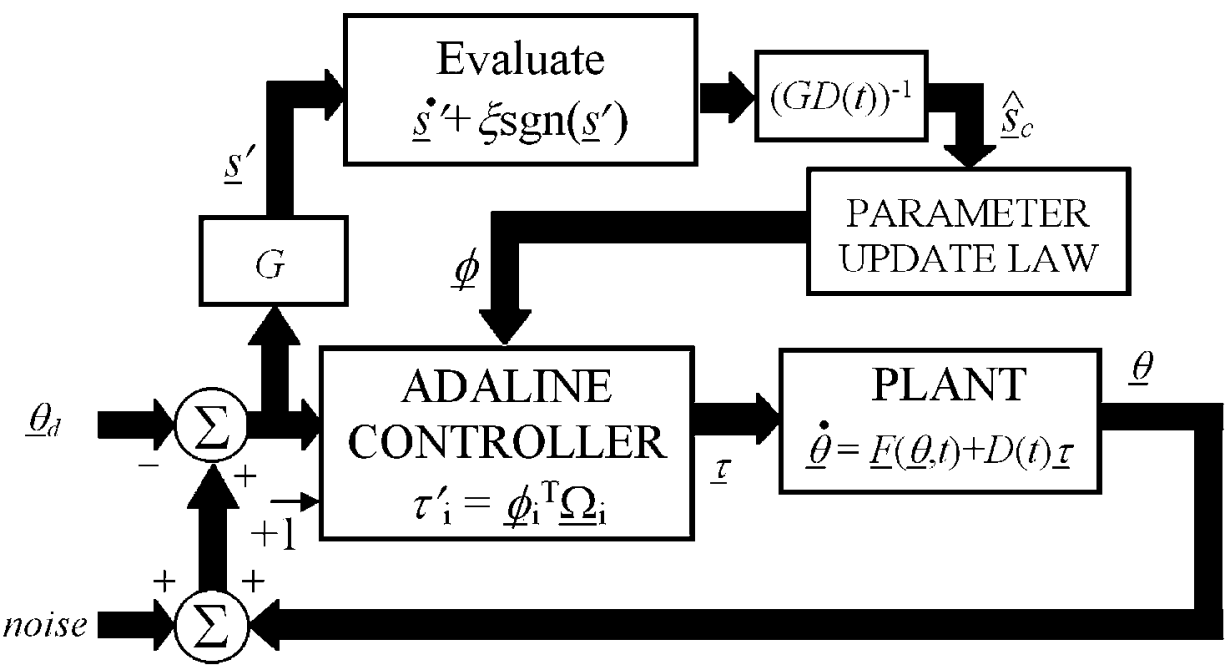

Figure 1 Block diagram of the control system 


$$
V(\underline{s})=\frac{1}{2^{\underline{S}}} \underline{\underline{S}}
$$

If the prescribed control signal satisfies $\breve{V}(\underline{s})=-\underline{s}^{\mathrm{T}} \xi$ sgn $(\underline{s})$, the negative definiteness of the time derivative of the Lyapunov function in (3) is ensured. In the above, $\xi$ is a positive definite diagonal matrix of dimension $n \times n$ and is a design

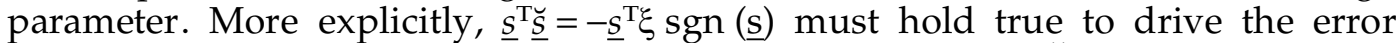
vector towards the sliding hypersurface. Utilizing $\breve{s}=-G \underline{\underline{\theta}}_{d}+G(\underline{F}(\underline{\theta}, t)+D(t) \underline{\tau})$ leads to the following control signal:

$$
\underline{\tau}^{*}=-(G D(t))^{-1}\left(G \underline{F}(\underline{\theta}, t)-G \underline{\underline{\theta}}_{d}\right)-(G D(t))^{-1} \xi \operatorname{sgn}(\underline{s})
$$

in which the first term is the equivalent control term and the second term is the corrective control term. For the existence of the components mentioned, the matrix
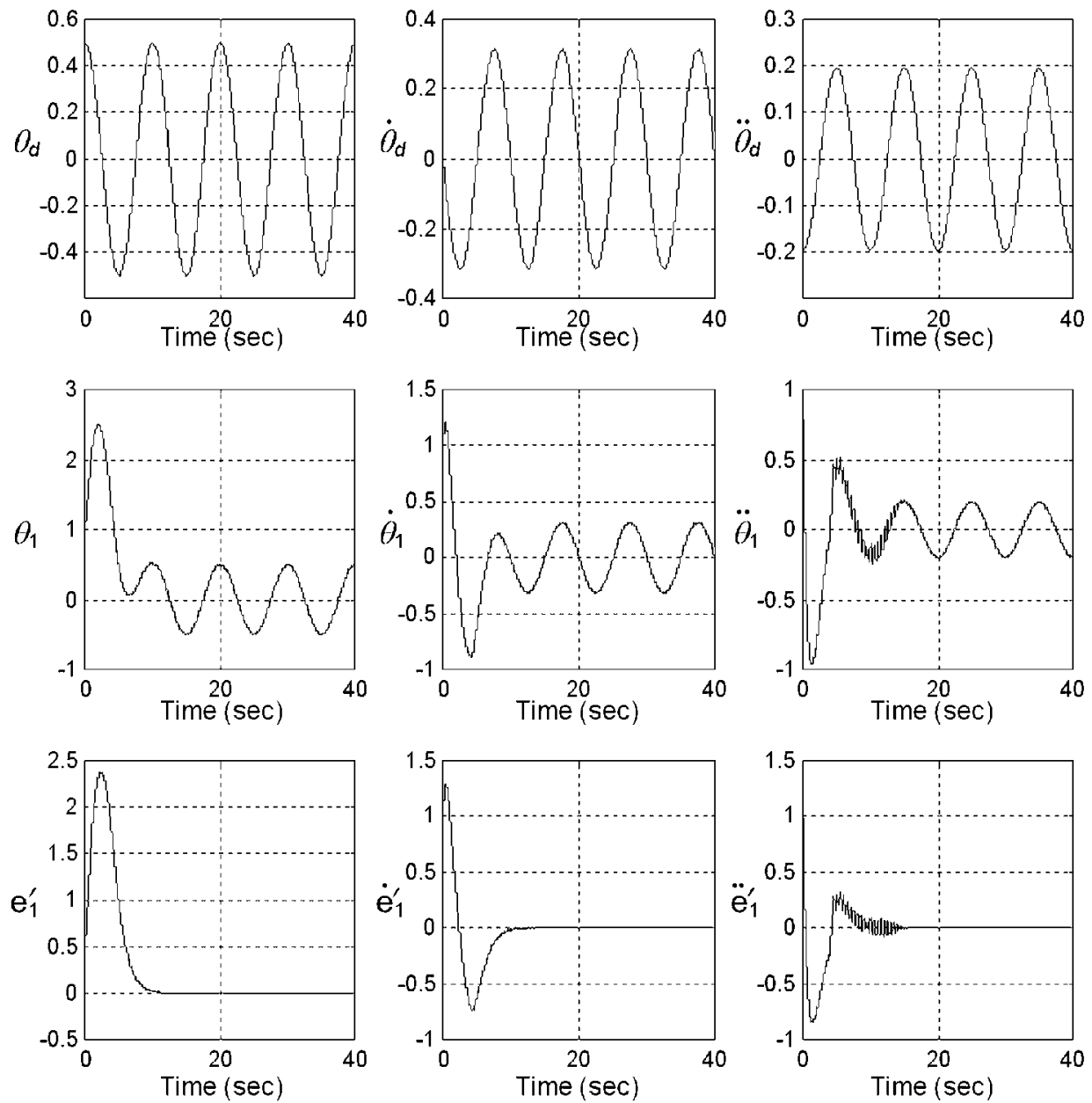

Figure 2 Reference trajectories, observed response and the error trends 

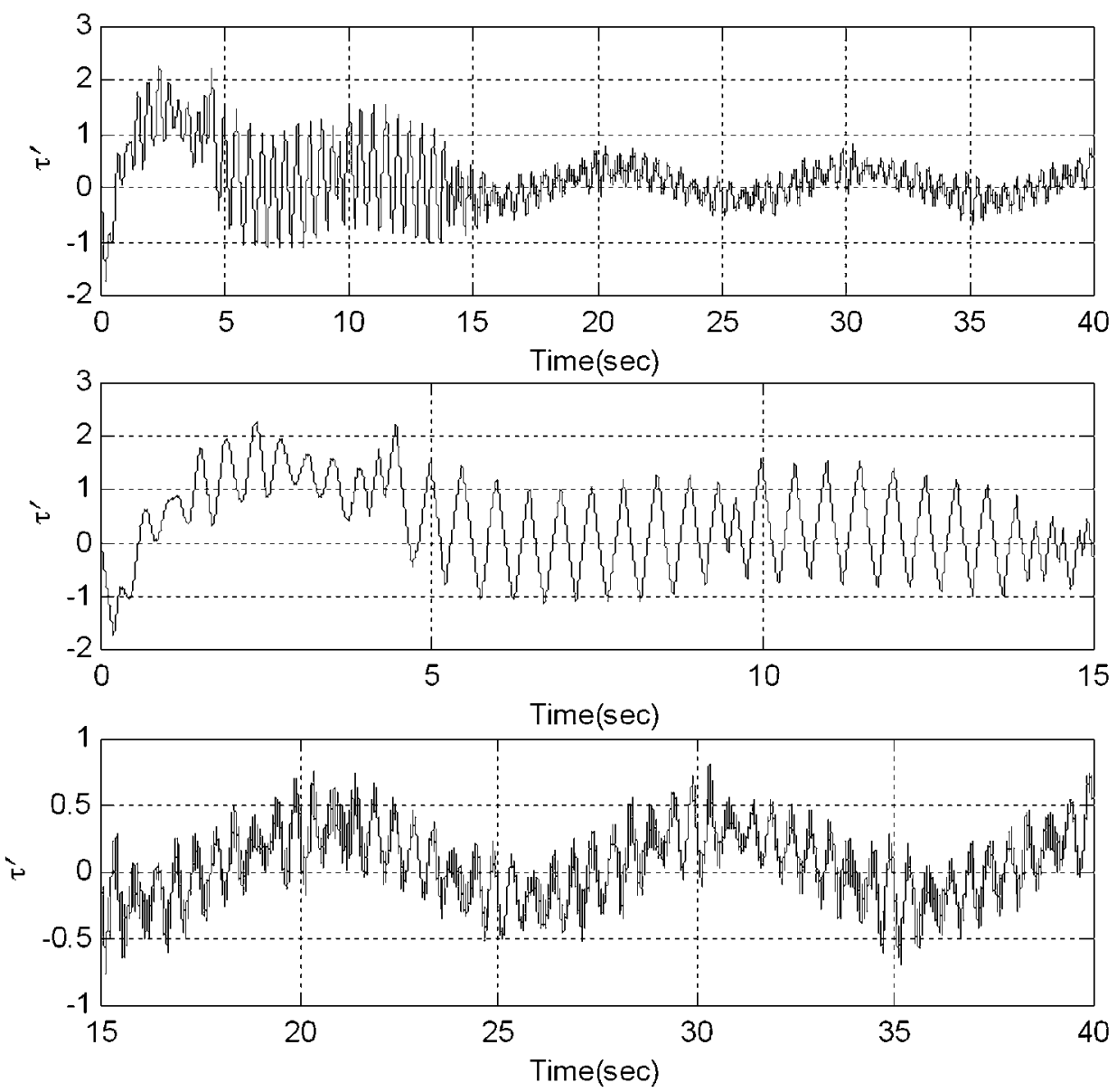

Figure 3 Applied control signal and its transient and steady state views

GD must not be rank deficient. In the literature, equivalent control is considered the low-frequency (average) component of the control signal. Because of the discontinuity on the sliding surface, the corrective term brings a high rate component (Utkin, 1992). If $\underline{e}(0)=\underline{0}$, the tracking problem can be considered as keeping $\underline{e}$ on the sliding surface; however, for nonzero initial conditions, the strategy must enforce the state trajectories towards the sliding surface, which is ensured by the negative definiteness of the time derivative of the Lyapunov function in (3). For the case of nonzero initial conditions, the phase until the error vector hits the sliding surface is called the reaching mode, the dynamic characteristics of the system during which are determined by the control strategy adopted. Application of the control input formulated in (4) imposes the dynamics described as $\underline{\breve{s}}=-\xi \operatorname{sgn}(\underline{s})$, which clearly enforces the error vector towards the sliding surface. Once the sliding surface is reached, the value of (2) becomes zero; and this enforces the error vector to move towards the origin due to the selection in (2). 
Aside from the practical difficulties of conventional SMC schemes, the control signal in (4) is computable if a nominal representation of the system under control is available. In what follows, a method for obtaining the error on the control signal is presented for unknown systems of structure (1).

\section{Calculation of the control error}

Denote the variables observed upon the application of (4) as the control signal with a superscript ${ }^{*}$, and denote those for any applicable control signal as primed. In other words, the control signal $\tau^{*}$ is the one creating the desired response, while $\tau^{\prime}$ is the signal produced by the controller and applied to the system. The goal is how $\underline{\tau^{\prime}} \rightarrow \underline{\tau}^{*}$ during the course of a control operation with $\underline{\theta}^{\prime}(0)=\underline{\theta}^{*}(0)$.

Clearly, when $\overline{\tau^{\prime}}$ is applied to the system of (1), the desired response must satisfy $\underline{s^{\prime}} \underline{\breve{s}^{\prime}}=-\underline{s^{\prime}} \bar{T} \xi$ sgn $\left(\underline{s^{\prime}}\right)$ in the closed loop. Equivalently, this requires the following:

$$
\underline{\breve{s}}^{\prime}=-G \underline{\underline{\theta}}_{d}+G\left(\underline{F}\left(\underline{\theta^{\prime}}, t\right)+D(t) \underline{\tau^{\prime}}\right)
$$

Since the command trajectory is same in both cases, solving $G \breve{\theta}_{d}$ from (4) and substituting the result into (5) yields

$$
\underline{\breve{s}^{\prime}}=G D(t)\left(\underline{\tau^{\prime}}-\underline{\tau}^{*}\right)+G\left(F\left(\underline{\theta}^{\prime}, t\right)-F\left(\underline{\theta}^{*}, t\right)\right)-\xi \operatorname{sgn}\left(\underline{s}^{*}\right)
$$

Defining $\underline{s}_{c} \triangleq \underline{\tau^{\prime}}-\underline{\tau}^{*}, \underline{\Delta F} \triangleq \underline{F}\left(\underline{\theta}^{\prime}, \mathrm{t}\right)-F\left(\underline{\theta}^{*}, \mathrm{t}\right)$ and adding to both sides of (6) results in (7).

$$
\underline{\breve{s}^{\prime}}+\xi \operatorname{sgn}\left(\underline{s}^{\prime}\right)=G D(t) \underline{s}_{c}+G \underline{\Delta F}-\xi\left(\operatorname{sgn}\left(\underline{s}^{*}\right)-\operatorname{sgn}\left(\underline{s^{\prime}}\right)\right)
$$

Several cross implications hidden in (7) can be clarified as follows:

$$
\begin{aligned}
& \underline{s}_{c} \rightarrow \underline{0} \Leftrightarrow \underline{\tau}^{\prime} \rightarrow \underline{\tau}^{*} \Leftrightarrow\left\{\begin{array}{c}
\left.(G D(t))^{-1} \underline{\underline{\underline{S}}^{\prime}}+\xi \operatorname{sgn}\left(\underline{s}^{\prime}\right)\right) \rightarrow \underline{0} \\
\& \\
(G D(t))^{-1}\left(G \underline{\Delta F}-\xi\left(\operatorname{sgn}\left(\underline{s}^{*}\right)-\operatorname{sgn}\left(\underline{s^{\prime}}\right)\right)\right) \rightarrow \underline{0}
\end{array}\right. \\
& \left.(G D(t))^{-1} \underline{\underline{s}}^{\prime}+\xi \operatorname{sgn}\left(\underline{s^{\prime}}\right)\right) \rightarrow \underline{0} \Leftrightarrow \underline{\tau^{\prime}} \rightarrow \underline{\tau}^{*} \Leftrightarrow\left\{\begin{array}{c}
\underline{s}_{c} \rightarrow \underline{0} \\
\& \\
(G D(t))^{-1}\left(G \underline{\Delta F}-\xi\left(\operatorname{sgn}\left(\underline{s}^{*}\right)-\operatorname{sgn}\left(\underline{s^{\prime}}\right)\right)\right) \rightarrow \underline{0}
\end{array}\right.
\end{aligned}
$$

Referring to (7) and the internal relations given in (8) and (9), assume that a biased estimate of $\underline{s}_{c} \triangleq \underline{\tau^{\prime}}-\underline{\tau}^{*}$ is computed as $\underline{\hat{s}}_{c}=(G D(t))^{-1}\left(\underline{\breve{s}^{\prime}}+\xi \operatorname{sgn}\left(\underline{s}^{\prime}\right)\right)$. Although $\underline{s}_{c}$ and $\hat{s}_{c}$ are slightly different in value, behavioral implication of both $\underline{\hat{s}}_{c} \rightarrow \underline{0}$ and $\underline{s}_{c} \rightarrow \underline{0}$ are the same, i.e., to observe $\underline{\breve{s}^{\prime}} \rightarrow \xi \operatorname{sgn}\left(\underline{s}^{\prime}\right)$. If an adaptation algorithm adjusts the parameters of a controller such that $\underline{\hat{s}}_{c} \rightarrow \underline{0}$, then due to above 
implications, $\underline{\tau^{\prime}} \rightarrow \underline{\tau^{*}}$ and $\underline{s}^{\prime} \rightarrow \underline{s}^{*}$. The designer must here notice that at each control cycle there will be an uncertainty on the estimated control error since $G \Delta F$ $-\xi\left(\operatorname{sgn}\left(\underline{s}^{*}\right)-\operatorname{sgn}\left(\underline{s}^{\prime}\right)\right) \neq \underline{0}$ during $\underline{s}^{\prime} \neq \underline{s}^{*}$, and the effect of this term is strictly dependent upon the vector function $\underline{F}(\underline{\theta}, t)$, which is unknown.

\section{Tuning of ADALINE controller parameters}

The input output relation of the $i$ th ADALINE controller is as $\tau_{i}^{\prime}=\underline{\phi}_{i}^{\mathrm{T}} \underline{\Omega}_{i}$, where $\underline{\Omega}_{i}=\left[e_{i}^{\prime} \breve{e}_{i}^{\prime} \ldots e^{\prime \prime} i_{i}^{\left(r_{i}-1\right)} 1\right]^{\mathrm{T}}$ is the vector of signals exciting the adjustable parameters denoted by $\phi_{i}$. In order not to be in conflict with the physical reality, the designer must impose $\left\|\underline{\phi}_{i}\right\| \leq B_{\Phi_{i^{\prime}}}\left\|\underline{\Omega}_{i}\right\| \leq B_{\Omega_{i}}\left|\tau_{i}^{*}\right| \leq B_{\tau_{i^{\prime}}^{*}}$ the truth of which state that the adjustable parameters of the controller, the time derivative of the signal exciting the adjustable parameter set and the time derivative of the target output of the controller remain bounded.

Theorem. For the $i$ th subsystem of the system described in (1), which is under the control loop shown in Figure 1, the use of an ADALINE controller having inputoutput relation $\tau_{i}^{\prime}=\underline{\phi}_{i}^{\mathrm{T}} \underline{\Omega}_{i}$ and the adaptation of the controller parameters as described in (10) enforces the value of the $i$ th component of the control discrepancy vector $\left(s_{c_{i}}\right)$ to zero:

$$
{\breve{\Phi_{i}}}_{i}=-\frac{\underline{\Omega}_{i}}{\underline{\Omega}_{i}^{\mathrm{T}} \underline{\underline{\Omega}}_{i}} k_{i} \operatorname{sgn}\left(s_{c_{i}}\right)
$$

where $k_{i}$ is a sufficiently large positive constant satisfying $k_{i}>B_{\Phi_{i}} B_{\Omega_{i}}+B_{\tau_{i}^{*}}$. The adaptation law in (10) drives an arbitrary initial value of $s_{c_{i}}$ to zero in finite time denoted by $t_{h i}$ satisfying the inequality in (11).

$$
t_{h_{i}} \leq \frac{\left|s_{c_{i}}(0)\right|}{k_{i}-\left(B_{\Phi_{i}} B_{\Omega_{i}}+B_{t_{i}^{*}}\right)}
$$

Proof: See Sira-Ramirez et al. (1995) and Efe et al. (2000).

An important feature of this approach is the fact that the controller parameters evolve bounded as assumed initially. The details of the bounded parametric evolution analysis can be found in Yu et al. (1998) and Efe et al. (2000).

Based on the discussion presented in the previous section, the adaptation algorithm uses the estimate of the control discrepancy vector, i.e., $\hat{s}_{c_{i^{\prime}}}$ as also illustrated in Figure 1.

\section{Practical issues}

The analysis and the design approach presented so far have tried to illuminate the SMC-based adaptation problem from a theoretical perspective. In this subsection, two important issues related to the practical applications of the discussed approach are highlighted. 


\subsection{Chattering}

Since the control decision is based on the sign of a quantity, which is very close to zero, the motion that is observed during the sliding regime exhibits highfrequency components, which is known as chattering. Referring to the control signal of (4), the second term is the well known corrective control action and is the primary reason for chattering. Several approximations can be utilized to avoid chattering at the cost of introducing a boundary layer. This paper uses the exact sign functions; however, an in-depth discussion on this issue can be found at Hung et al. (1993) and Slotine and Li (1991).

\subsection{Actuation speed}

Another important issue is the actuation speed of the system under control, i.e., the ability to respond to what is imposed timely. Since the details concerning the dynamic model of the plant under control are assumed to be unavailable, what causes a difficulty from a practical point of view is the selection of the design matrix $\xi$, which characterizes the behaviour during the reaching mode. The values of this quantity can only be set by trial-and-error, due to the lack of systemspecific details.

\section{An illustrative example}

This section demonstrates the performance of the proposed scheme for a thirdorder system studied previously by Roy and Olgac (1997) and Yilmaz and Hurmuzlu (2000). The dynamic equation describing the system is given in (12).

$$
\begin{array}{r}
\theta^{(3)}=-0.5 \theta-0.5 \breve{\theta}^{3}-0.5 \ddot{\theta}|\ddot{\theta}|+(1+0.1 \sin (\pi t / 3)) \tau+\kappa_{1}(t)+\kappa_{2}(t)+ \\
(-0.05+0.25 \sin (5 \pi t)) \theta+(-0.03+0.3 \cos (5 \pi t)) \breve{\theta}^{3}+(-0.05+0.25 \sin (7 \pi t)) \ddot{\theta}|\theta ̈|
\end{array}
$$

where $\kappa_{1}(t)=0.2 \sin (4 \pi t)$ is the disturbance used in Roy and Olgac (1997) and Yilmaz and Hurmuzlu (2000), and $\kappa_{2}(t)$ is the zero mean Gaussian noise corrupting the state information to be used by the controller additively. The standard deviation for all three noise components is equal to 0.00025 and the noise sequences are within \pm 0.001 interval, with probability very close to unity.

The work presented by Roy et al. assumes that the nominal system dynamics is known and the uncertain part is comprised of what is given as the last three terms in (12). The primary difference between what has been discussed so far and what is assumed in Roy and Olgac (1997) should be stressed, as the approach we discuss only assumes the achievability of the SMC task, hence the uncertainties are represented in the system dynamics, whose form is known but the details are not. As the controller, a four-input single-output ADALINE structure is used with input vector $\Omega_{i}=\left(e_{i}^{\prime} \breve{e}_{i}^{\prime} \ddot{e}_{i}^{\prime} 1\right)^{\mathrm{T}}$. The tuning is performed on all four parameters, which are initially set to zero. Since the system comprises a single subdynamics, which is of order $3, n=1$ and $r_{1}=3$. The system is under the feedback loop as 


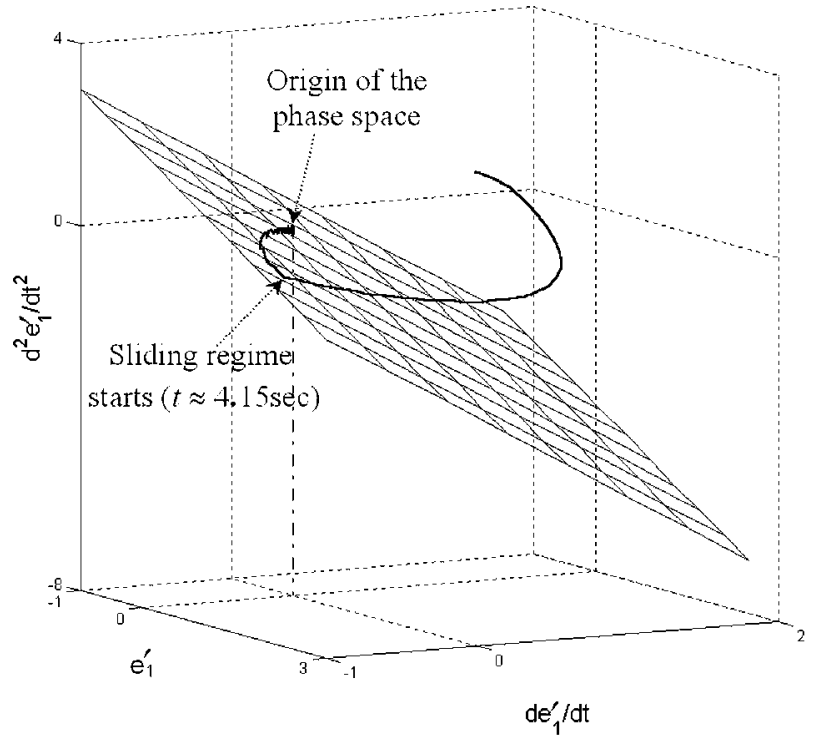

Figure 4 Sliding surface and the motion in the phase space

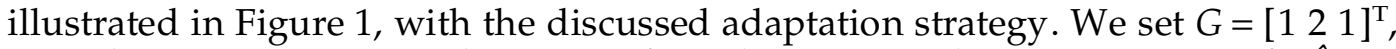
i.e., $\lambda_{1}=1, k_{1}=10$ and $\xi=1$, for the SMC design matrix of $\hat{\underline{s}}_{c}=$ $(\mathrm{GD}(\mathrm{t}))^{-1}\left(\underline{\breve{s}^{\prime}}+\xi \operatorname{sgn}\left(\underline{s}^{\prime}\right)\right)$.

Parallel to Roy and Olgac (1997), the reference state trajectory described as $\theta_{d}=0.5 \cos (\pi t / 5)$ is used in the simulations with $\breve{\theta}_{d}(0)=0, \ddot{\theta}_{d}(0)=-\pi^{2} / 50$. The simulation step size is $0.5 \mathrm{~ms}$. Initially, the states of the system have the following values, $\theta(0)=1, \breve{\theta}(0)=1$ and $\ddot{\theta}(0)=1$. In the first column of Figure 2, the reference state trajectory for $\theta$ state, observed system response in $\theta$ and the discrepancy between them are illustrated. The second and the third columns of this figure illustrate the responses relevant to $\breve{\theta}$ and $\ddot{\theta}$ states. From the bottom row of this figure, convergent error trends are clear for all three error variables.

In the top row of Figure 3, the applied control signal is illustrated. In order to see the transient behaviour in detail, the middle row depicts the same signal for the first $15 \mathrm{~s}$ and the bottom row shows the behaviour after $t=15 \mathrm{~s}$. Apparently, the applied control signal settles down to a steady-state solution after a reasonably fast transient, which takes place during the first $15 \mathrm{~s}$. One important note here should be on the selection of the design parameter $\lambda$. The value is taken as 5 in Roy and Olgac (1997); however, we use $\lambda=1$, because the behaviour with this value results in a much better system response. Figure 4 illustrates the trajectory followed in the phase space. The error vector hits the sliding surface first around $t \approx 4.15 \mathrm{~s}$, and continues moving on it as enforced by the algorithm. Next, the behaviour in the parameter space should be taken into consideration. As seen in Figure 5, the first three parameters settle down to constant solutions as their multipliers are the convergent error terms, and the fourth one, whose multiplier is a constant bias unity, continuously provides the necessary control input during the motion around the origin of the phase space. 

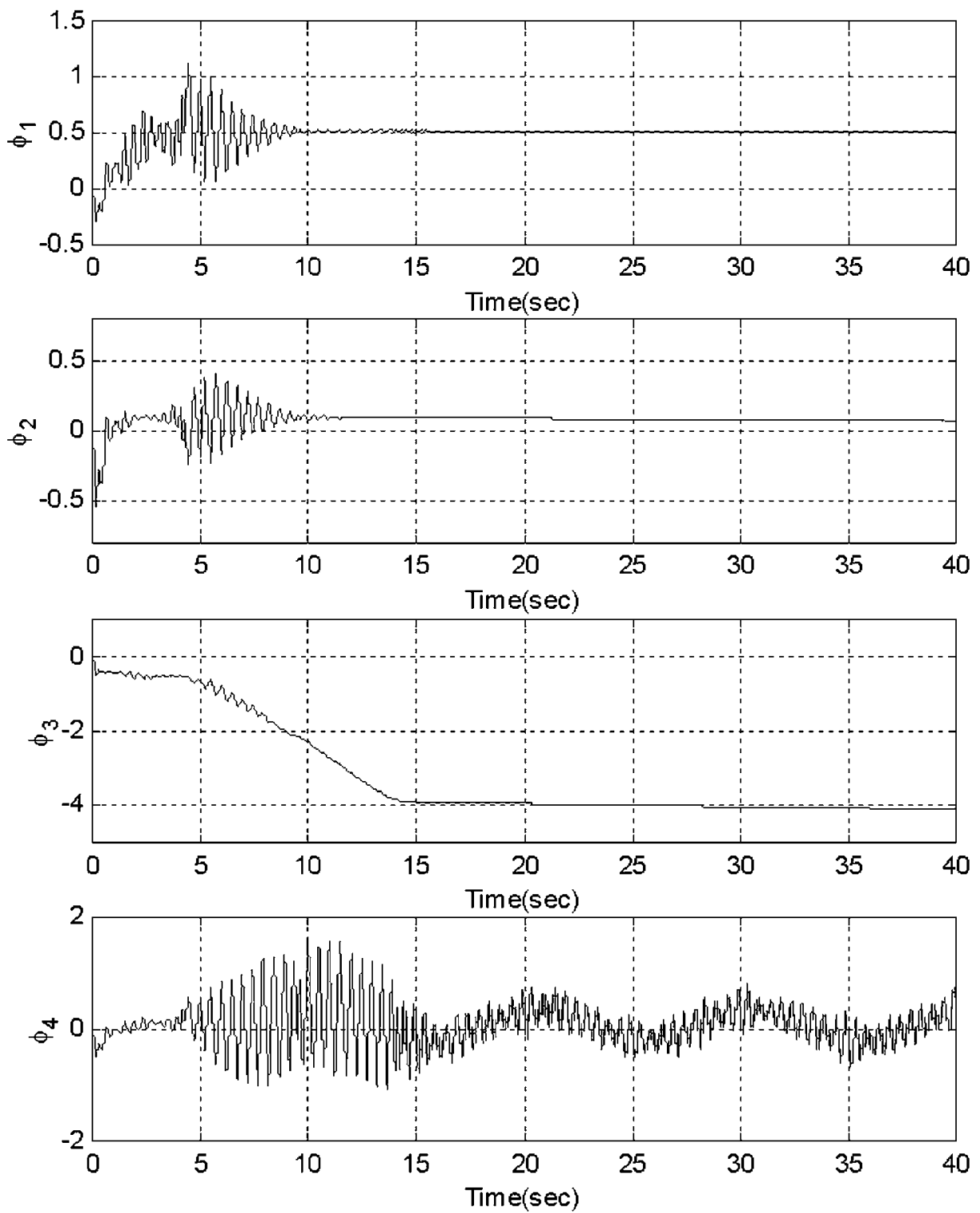

Figure 5 Time evolution of the adjustable controller parameters

If $\underline{F}(\underline{\theta}, t)$ of (1) were known perfectly, the target response obtained through the construction of the target control signal by using (4) would be compared as depicted in Figure 6. Clearly, the Euclidean norm of the difference between the two error vectors, namely $\left\|\underline{e}^{\prime}-\underline{e}^{*}\right\|$, converges to zero rapidly. The trajectories followed in the phase space also demonstrate that the target regime is slightly different from that achieved by the discussed method since $G \underline{\Delta F}-\xi\left(\operatorname{sgn}\left(\underline{s}^{*}\right)-\operatorname{sgn}\left(\underline{s}^{\prime}\right)\right)$ 

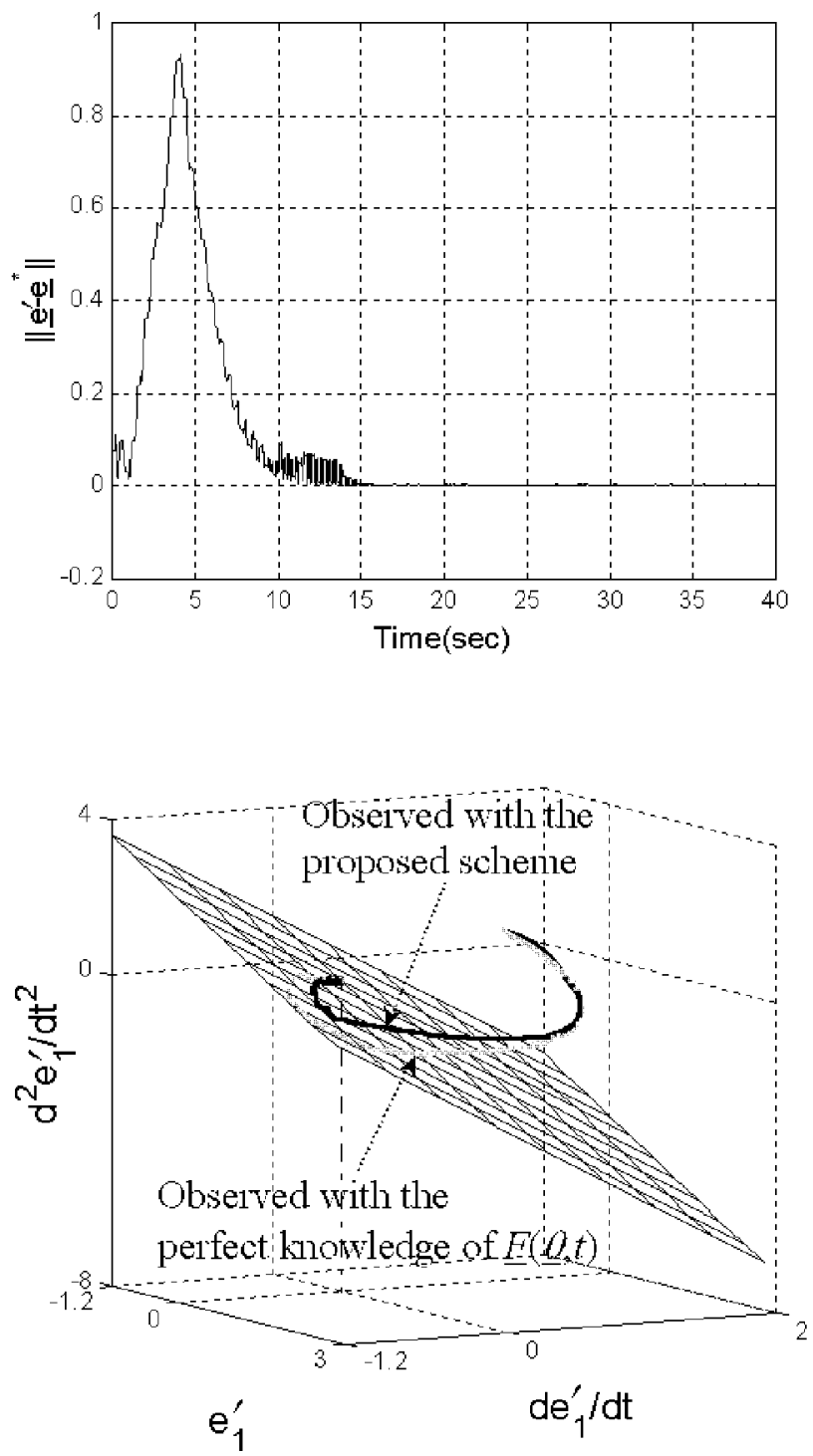

Figure 6 Comparison of the proposed approach $(\underline{F}(\underline{\theta}, t)$ is unknown) and the result of the traditional scheme when $\underline{F}(\theta, t)$ is known perfectly

of (7) cannot be constructed numerically. This comparison justifies the creation and maintenance of the SMC task claim of the paper.

Lastly, we analyse the computational burden of the algorithm. The required number of floating point operations (flops) to perform the inner product of $\Omega_{i}$ and $\phi_{i}$ is equal to 9 for a third-order system and that to tune the parameters is equal to 63 flops. A total of 72 flops with two comparisons are performed and this result is highly affordable even for the average-speed microcontrollers. 


\section{Conclusions}

This paper discusses the design of an ADALINE-based variable structure control strategy for a class of systems. The approach assumes that the SMC task is achievable and the system has the structure given in (1). A number of issues in SMC design are discussed from the point of control engineering and a case study has been presented. It is shown that the tuning strategy meets the desired specifications. Furthermore, the control system retains a high degree of robustness against disturbances and uncertainties. A major contribution of the paper is the creation and maintenance of a predefined sliding regime without knowing the analytical details describing the plant dynamics. The acceptable smoothness of the produced control signal and the computational simplicity of the approach are the prominent features recommending the use of the proposed adaptation law. The potential application field of the scheme presented covers all controller structures, whose outputs are linear in the adjustable parameters. Therefore, if the task is well specified, the removal of the ambiguity on the control signal error for intelligent control applications has been shown to be achievable without externally identifying the system under investigation.

\section{Acknowledgements}

The author gratefully acknowledges the constructive suggestions of the anonymous reviewers.

\section{References}

Efe, M.O., Kaynak, O. and Yu, X. 2000: Sliding mode control of a three degrees of freedom anthropoid robot by driving the controller parameters to an equivalent regime. Transactions of the ASME: Journal of Dynamic Systems, Measurement and Control 122, 632-40.

Hsu, L. and Real, J.A. 1997: Dual mode adaptive control using gaussian neural networks. Proceedings of the 36th Conference on Decision and Control, (CDC), New Orleans, Louisiana, 4032-37.

Hsu, L. and Real, J.A. 1999: Dual mode adaptive control. Proceedings of the IFAC'99 World Congress, K, Beijing, China, 333-37.

Hung, J.Y., Gao, W. and Hung, J.C. 1993: Variable structure control: a survey. IEEE Transactions on Industrial Electronics 40, 1-9.

Kaynak, O., Erbatur, K. and Ertugrul, M. 2001: The fusion of computationally intelligent methodologies and sliding-mode control - a survey. IEEE Transactions on Industrial Electronics 48, 4-17.
Parma, G.G., Menezes, B.R. and Braga, A.P. 1998: Sliding mode algorithm for training multilayer artificial neural networks. Electronics Letters 34, 97-98.

Roy, R.G. and Olgac, N. 1997: Robust nonlinear control via moving sliding surfaces $n$-th order case. Proceedings of the 36 th Conference on Decision and Control, San Diego, California, 943-48.

Sanner, R.N. and Slotine, J.J.E. 1992: Gaussian networks for direct adaptive control. IEEE Transactions on Neural Networks 3, 837-63.

Sira-Ramirez, H. and Colina-Morles, E. 1995: A sliding mode strategy for adaptive learning in adalines. IEEE Transactions on Circuits and Systems - I: Fundamental Theory and Applications 42, 1001-12.

Sira-Ramirez, H., Colina-Morles, E. and Rivas-Echevverria, F. 2000: Sliding modebased adaptive learning in dynamical-filterweights neurons. International Journal of Control 73, 678-85. 
Slotine, J.J.E. and Li, W. 1991: Applied nonlinear control. Englewood Cliffs, New Jersey: Prentice-Hall.

Utkin, V.I. 1992: Sliding modes in control optimization. New York; Springer Verlag.

Yilmaz, C. and Hurmuzlu, Y. 2000: Eliminating the reaching phase from variable structure control. Transactions of the ASME, Journal of Dynamic Systems, Measurement and Control $122,753-57$.

Yu, X., Zhihong, M. and Rahman, S.M.M. 1998: Adaptive sliding mode approach for learning in a feedforward neural network. Neural Computing $\mathcal{E}$ Applications 7, 289-94. 